\title{
Carnap on logic and rationality
}

\author{
Georg Schiemer
}

This is a post-peer-review, pre-copyedit version of an article published in [Synthese]. The final authenticated version is available online at: http://dx.doi.org/[10.1007/s11229-016-1252-4].

Rudolf Carnap is undoubtedly one of the central figures in twentieth-century scientific philosophy. While his work covers a wide spectrum of topics in philosophy of logic and mathematics, philosophy of language, formal philosophy of science, and probability theory (among other fields), a particular method of doing philosophy can be identified in all of his contributions. This is the use of formal logic in his approach to philosophical problems. Logic plays an essential role in Carnap's work throughout his intellectual career, from his early type-theoretic formulation of a constitution theory of knowledge in Der logische Aufbau der Welt (Carnap 1928) to his mature work on a theory of probability, e.g. (Carnap 1950).

The importance of logical analysis and explication in Carnap's thought and its influence on subsequent philosophical developments have been widely acknowledged in recent scholarship. ${ }^{1}$ Less attention has so far been dedicated to Carnap's own contributions to formal logic and its metatheory. Thus, he not only applied logical methods in his philosophical work, but also constructed new logical systems and languages along the way. Moreover, there has also been a continuous philosophical reflection on the logical methods used by him. This includes systematic considerations about the nature of logic and its normative status, its proper role in scientific reasoning, as well as its relation to other formal disciplines, most importantly mathematics.

The present special issue is dedicated to a novel assessment of Carnap's contributions to logic and of the different uses of logical methods in his philosophical work. The volume comprises novel research articles on different aspects of Carnap logical work

Department of Philosophy, University of Vienna, Universitätsstraße 7, 1010 Vienna, Austria \& Munich Center for Mathematical Philosophy, LMU Munich, Geschwister-Scholl-Platz 1, D-80539 Munich, Germany

E-mail: georg.schiemer@univie.ac.at

1 See, for instance, (Carus 2007; Friedman 1999; Friedman and Creath 2007; Frost-Arnold 2013; Wagner 2009; Reck 2004) and further reference provided there. 
from different phases in his intellectual career. The articles were originally presented at the conference "Carnap on Logic" held at the Munich Center for Mathematical Philosophy at LMU Munich in June 2013. ${ }^{2}$ The central objective of the conference was to bring together a number of leading Carnap scholars and to address the following questions:

- What are Carnap's main contributions to logic and metalogic?

- How did Carnap's conception of logic change over the course of his intellectual career?

- What are the central tenets of Carnap's philosophy of logic? How are his views related to contemporary debates, e.g. on the characterization of logical constants or the normativity of logic?

- What is Carnap's methodology of logical formalization? How precisely did he apply logic in philosophy?

Several contributions in this special issue focus on the first question and investigate Carnap's early work on higher-order logic, specifically on Russell's \& Whitehead's type-theory from Principia Mathematica as well as Carnap's early contributions to the metatheory of axiomatic theories in his "general axiomatics" project ((Carnap 1927), (Carnap 1928/2000)). A second and related focus here concerns the immediate intellectual background of Carnap's logical work. The aim here is to further clarify how his contributions were influenced by figures like Frege, Russell, Fraenkel, Wittgenstein, Tarski, Gödel, and Kemeny (among others).

Research on Carnap's philosophy of logic in this volume focuses on a number of issues. One aspect studied here concerns Carnap's pre-Syntax conception of logic and the question whether this is correctly described as belonging to the "universalist" (as opposed to the "model-theoretic") tradition in logic. ${ }^{3}$ A related issue is Carnap's understanding of formal languages and their model-theoretic semantics: several articles address the question how his views changed between his early work on Principia Mahematica-style languages and the treatment of formal languages in later contributions up to the Schilpp volume (Carnap 1963). A second focus concerns Carnap's logical pluralism first articulated in Logical Syntax of Language (Carnap 1934). Several articles in the volume analyze the implications of Carnap's "principle of tolerance" for his understanding of the normativity of logic and the rationality of reasoning more generally.

Regarding the last two questions, several articles retrace Carnap's attempts to explicate concepts such as logical truth and analyticity, different notions of completeness for axiomatic theories, empirical significance, rationality, etc. Moreover, it is also discussed which principles or desiderata should guide the general method of logical explication according to Carnap.

\footnotetext{
2 For further information on the conference and its participants see the conference website www.carnaponlogic2013.philosophie.uni-muenchen.de.

3 For such an interpretation, see, for instance, (Coffa 1991) and (Hintikka 1992).
} 
The volume comprises eleven contributions on these topics, including ten original research articles as well as a transcription and English translation (given by André Carus) of Carnap's notes on "Value concepts" from 1958. In the following sections, we will briefly survey these contributions and indicate some thematic points of contact between them.

\section{Early metalogic, invariance, and logicality}

Several articles in this volume focus on Carnap's contributions to logical metatheory and his philosophy of logic before Logical Syntax. The articles by Loeb and Schiemer, Zach \& Reck both deal with Carnap's early work on "general axiomatics" in his manuscript Untersuchungen zur Allgemeinen Axiomatik (Carnap 1928/2000) written around 1928.

Iris Loeb's "The role of universal language in the early work of Carnap and Tarski" gives a comparative study of Tarski's The concept of truth in formalized languages (Tarski 1935) and Carnap's Untersuchungen. The starting point in her paper is a much discussed idea in the history of logic, namely the distinction between two opposing traditions in modern logic, the 'universal' and the 'model-theoretic' tradition. ${ }^{4}$ Loeb specifies the former tradition with respect to Tarski's work by distinguishing between two senses of logical universality. She dubs these the semantic and the syntactic universality of a logical language. According to the second notion, a language is universal if all other formalized languages can be included as fragments of it.

Loeb argues that both in Tarski's treatment of formal languages and in Carnap's use of type-theoretic logic in his study of axiomatic theories, this notion of syntactic universality is assumed. Specifically, in Untersuchungen, Carnap works within a "basic discipline", viz. a universal logical language in the above sense, in which different axiomatic theories are formalized..$^{5}$ The axiom systems are formulated as fragments of this basic discipline, that is, as propositional functions with a number of free variables expressing its primitive terms.

The article also gives a closer comparison between Carnap's and Tarski's respective metatheoretic approaches. While there is a direct connection between the two accounts regarding the assumption of a universal language that allows one to study its own fragments, there are also important conceptual differences. The main one, Loeb argues, is how such language fragments are conceived: in Tarski's work on truth, the object language (understood as a sublanguage of the metalanguage) is closed under derivation rules. In contrast, in Carnap's case, axiomatic theories are also treated as fragments of the basic discipline, but they remain open fragments, that is, not closed under such rules. As a consequence of this, Loeb argues, Carnap is forced

\footnotetext{
4 See (van Heijenoort 1967) and (Goldfarb 1979) for a closer discussion of these two logical traditions.

5 Compare, in particular, (Awodey and Carus 2001) for a detailed discussion of Carnap's approach.
} 
to adopt a substitutional account of models of the axiomatic theories studied in his manuscript.

Loeb finally retraces the mathematical background of both Tarski's and Carnap's works. In Carnap's case, this is the development of modern formal axiomatics in nineteenth-century und early twentieth-century mathematics (as developed in work by Dedekind, Hilbert, and the Peanists, among others). In contrast, Loeb argues, it would be wrong to identify Tarski's metatheoretical work from the 1930s with this tradition of modern axiomatics: at least in Tarski (1935), Tarski still upheld a more classical, "contentual" understanding of axiomatic theories.

In “Carnap's early metatheory: scope and limits”, Georg Schiemer, Richard Zach \& Erich Reck provide a critical reassessment of Carnap's work on general axiomatics, in particular of his attempts to logically explicate several metatheoretical notions of completeness in Untersuchungen. The article builds on previous scholarly work (e.g. (Awodey and Carus 2001) and (Reck 2004)) and focuses on three central differences between Carnap's approach and modern metalogic: (i) the fact that in (Carnap 1928/2000) a clear distinction between object language and metalanguage is still missing; (ii) the missing separation between a syntactic (or proof-theoretic) and a semantic (or model-theoretic) study of theories; and (iii) Carnap's understanding of logical languages as meaningful formalisms (as opposed to the modern understanding as formal languages).

The paper first surveys Carnap's specific method of formalizing axiomatic theories and their models in a type-theoretic framework as well as his definitions of different notions of completeness (i.e. non-forkability, monomorphism, decidability, etc.). Carnap's attempt to explicate these concepts in higher-order logic clearly differs from the modern approach. In particular, whereas notions such a categoricity or semantic completeness are nowadays formulated in a separate meta-language, Carnap expresses them in a single and fully interpreted logical language, i.e. his "basic system". Sections 3 and 4 of the article give a modern reconstruction of this 'monolinguistic' approach. In particular, it is shown how the kind of model-theoretic domain variation built into these metatheoretic concepts is simulated in Carnap's type-theoretic approach.

Given this, Schiemer, Zach \& Reck turn to a discussion of the implications of Carnap's omission of a clear-cut distinction between proof-theoretic concepts (such as derivability) and semantic concepts (such as truth in a structure or logical consequence) in his axiomatics project. As is shown, this issue becomes particularly relevant in his so-called Gabelbarkeitssatz, a metatheorem stating the equivalence between the notions of non-forkability, monomorphism, and decidability. The paper finally addresses Carnap's constructivist philosophy of mathematics in Untersuchungen. This is reflected in his distinction between two ways to understand the completeness properties discussed above, namely as "absolute" or "constructive" concepts in general axiomatics. The authors give a closer analysis of these notions and discuss how Carnap's views are related to those of his contemporaries Hilbert, Bernays, and Ackermann. 
While the thematic focus of the first two articles is on Carnap's early contributions to logical metatheory, Steve Awodey's and Richard Creath's contributions are mainly concerned with Carnap's philosophy of logic. More specifically, both articles discuss his attempt to provide a precise characterization of logical expressions and logical truth in (Carnap 1934). Steve Awodey's “Carnap and the invariance of logical truth" focuses on a general idea underlying Carnap's work which Awodey calls the "invariance conception of logical truth". Logical constants are specified in Logical Syntax in terms of the notion of the determinateness of logical statements. More precisely, in $\S 50$ of (Carnap 1934), the set of logical symbols of a given language is defined as the largest set of symbols such that every sentence formed exclusively in terms of these symbols is determinate, i.e. its truth or falsity can be established solely on the basis of the transformation rules of the language. As Awodey shows, this attempt to demarcate between the logical and descriptive vocabulary of a language reflects a more general approach to logicality in terms of invariance conditions at work not only in the contributions of Carnap and Tarski but also in more recent work in philosophy of logic and the foundations of mathematics. In Carnap's Logical Syntax, this is the idea that the truth values of logical statements are invariant under reinterpretations of the descriptive or empirical terms of the language in question.

The paper argues for two claims: the first one is that the "invariance conception of logical truth" forms a unit idea that occurs at different stages in Carnap's work on logic: there is a pre-Syntax version of it, formulated in Carnap's project on general axiomatics from the late 1920s. In particular, the notion of "formal" or "structural" properties of models is defined in (Carnap 1928/2000) in terms of the invariance of properties under isomorphic transformations. As Awodey points out, this idea of specifying such properties in terms of isomorphism invariance was transformed in Logical Syntax into a purely syntactic invariance criterion, namely the determinacy of statements in terms of (syntactic) transformation rules.

The second claim defended in the article also relates to this shift in Carnap's work from a semantic structuralism to a syntactic formalism. It concerns a general objection against Carnap's definition of logical truth in Logical Syntax raised by a number of scholars, most importantly Goldfarb, Ricketts, and Friedman. ${ }^{6}$ Very roughly, the objection states that Carnap's determinacy criterion for logical expressions is incompatible with his principle of logical tolerance. This is due to the fact that Carnap's criterion seems to presuppose a powerful metalanguage in which each statement (or class of statements) of the object language in question is decidable. As Awodey argues, this objection falsely rests on the identification of Carnap's notion of logical determinacy with the stronger notion of decidability: a statement is determinate according to Carnap if its truth value remains stable under all possible interpretations of its non-logical vocabulary. In turn, it is decidable if one can effectively specify its truth value relative to a given interpretation. Since the requirements on the richness of the metalanguage are relatively weak in order to establish the determinacy of a

${ }^{6}$ A detailed list of references relevant here is given in Awodey's paper. 
statement, Awodey holds that reading Carnap's account of logical truth in the former way does not result in any conflict with his logical conventionalism.

Awodey's article finally mentions several points of contact between Carnap's use of invariance criteria for logical truth and more recent attempts in philosophy of logic to characterize logical constants in terms of invariance under structure-preserving permutations (of a domain) or mappings (between models). He also points to a connection between Carnap's approach and recent work on the univalent foundations project. It is shown that a central axiom in this project, Voevodsky's Univalence Axiom expresses a similar invariance condition for mathematical objects: it states that two objects that are homotopy equivalent, i.e. whose properties are invariant under homotopy equivalence, are to be identified.

Richard Creath's "The logical and the analytic" develops a novel analysis of Carnap's work on logical expressions in Logical Syntax. The paper has two thematic parts. In the first one, Creath gives a detailed discussion of Carnap's general definition of logical expressions in section 50 of (Carnap 1934) as well as of several objections raised against it in the subsequent literature. Carnap's definition is the following one:

Let $K_{1}$ be the product of all expressional classes $K_{i}$ of $S$ which fulfill the following four conditions:

1. If $A_{1}$ belongs to $K_{i}$, then $A_{1}$ is not empty and there exists a sentence which can be sub-divided into partial expressions in such a way that all belong to $K_{i}$ and one of them is $A_{1}$.

2. Every sentence which can be thus subdivided into expressions of $K_{i}$ is determinate.

3. The expressions of $K_{i}$ are as small as possible, that is to say, no expression belongs to $K_{i}$ which can be sub-divided into several expressions of $K_{i}$.

4. $K_{i}$ is as comprehensive as possible, that is to say, it is not a proper sub-class of a class which fulfils both (1) and (2).

An expression is called logical $\left(A_{l}\right)$ if it is capable of being sub-divided into expressions of $K_{1}$; otherwise it is called descriptive $\left(A_{d}\right)$. (Carnap 1934, pp. 177-178)

Creath first discusses several objections against this definition raised in (Mac Lane 1938) as well as in Quine's "Carnap and Logical Truth" (Quine 1960). Mac Lane's objections are based on counter-examples of expressions that are intuitively logical but turn out as descriptive according to Carnap's account. They concern, in particular, the maximality clause (4) in Carnap's definition. Creath formulates several modifications of the conditions in the definiens that rule out these counterexamples. Quine's main objection concerns the question whether the addition of non-logical terms such as "is heavier than" to a class of determinate statements will qualify as logical in Carnap's account. Creath shows, based on a number of examples, that this is not the case. Thus, Quine's claim that the addition of such terms to the logical terms will preserve determinacy is shown to be wrong.

Based on this, Creath goes on to investigate additional objections against Carnap's definition that have been formulated in more recent work. These include a counterexample based on the use of an empirical predicate $F$ (standing for "has a mass of one kilogram") and an atomic formula of the form $F a$ (where $a$ is a name of the International Prototype). This is clearly an ostensive definition of the concept of mass and 
therefore is determinate in Carnap's sense. As is shown, this and similar counterexamples are ruled out in a more recent proposal to modify Carnap's syntactic criterion of logicality given in (Bonnay 2009). Nevertheless, Creath argues, Bonnay's revised definition is "only partially successful": a specific problem that Creath sees here is that the new criterion of logical constants is built on the notion of names which remains undefined in Carnap's "General Syntax" section of (Carnap 1934).

In the second, more philosophical part of the article, Creath analyzes the impact of Carnap's lack of a working definition of logical expressions for his general project of clarifying the notion of analyticity. Creath's main point here is that this fact is not as fatal to Carnap's project as one would expect. This is due to the fact that in his subsequent work on semantic systems, most importantly in his Series of Semantics monographs from the 1940s, the semantic notion of analyticity (understood now as truth in virtue of the semantic rules of a system) is no longer dependent on a principled distinction between logical and non-logical (or descriptive) terms.

\section{Logical truth, analyticity, and pure semantics}

Creath's discussion of Carnap's post-Syntax work on the notion of analyticity is directly connected to both Pierre Wagner's and Peter Olen's articles in this volume. Both authors focus on different aspects of Carnap's contributions to semantics throughout the 1940s.

Pierre Wagner's “Carnapian and Tarskian semantics” offers a new assessment of the relevance of Tarski's work on scientific semantics - in particular on formal truth and logical consequence (Tarski 1935, 1936)_for Carnap's own 'semantic turn', i.e. his move from a purely syntactical to a semantic study of logical and scientific language systems. Wagner's primary aim is to put into perspective the widespread assumption that Carnap readily and wholeheartedly adopted Tarskian semantics after the publication of Logical Syntax. In particular, Wagner emphasizes that one needs to distinguish between different stages in Carnap's work on semantics: there is a first period, starting with Carnap's monograph Foundations of Logic and Mathematics (Carnap 1939) and continuing until the first book in the Series in Semantics, namely Introduction to Semantics (Carnap 1942). The second period comprises Carnap's work on state descriptions in modal and inductive logic, in particular in Carnap (1947) and (Carnap 1950). The final stage, starting in 1955 with Carnap's manuscript Notes on semantics, is characterized by his work on model-theoretic concepts.

Having outlined these different phases in Carnap's intellectual career, Wagner argues for two interpretive claims: The first one is that Carnap's conception of semantics in each of these stages differs significantly from his previous accounts. The second point is that each of these stages shows fundamental differences between Carnap's views and those of Tarski. It is argued in the paper that Tarski's effective influence on Carnap own work on semantics was in fact minimal. For instance, a central distinction in Carnap's semantic program is that between factual truth and logical truth (or $L$-truth). In this point, Carnap's account differs fundamentally from Tarski's: whereas 
Tarski doubted whether a clear-cut distinction between factual or descriptive truth and logical truth could meaningfully be drawn, this distinction remains at the heart of Carnap's enterprise of specifying the semantic structure of scientific languages.

A related aspect discussed in Wagner's article is that, at least from 1942 onward, Carnap developed several systems of semantics that are clearly not based on Tarski's previous metatheoretical work. This concerns in particular, Carnap's different attempts to explicate the notion of $L$-truth in terms of the notion of the "range" of a statement (first defined in (Carnap 1947) as the class of "state-descriptions" in which a statement holds). As Wagner shows, this and related semantic concepts have not connection to Tarski's scientific semantics, but are rather motivated by previous work of Wittgenstein and Waismann.

Peter Olen's "A forgotten strand of reception history: understanding pure semantics" also deals with the transition in Carnap's work from a purely syntactic study of formal languages to a study of semantic systems. The focus here is on the reception of Carnap's treatment of semantic notions in (Carnap 1942) in work by Gustav Bergmann and Everett Hall. Olen's paper consists of three parts: in the first part, he surveys Bergmann's and Hall's critical discussions of Carnap's pure semantics, in particular his rules of designation and truth. On Hall's interpretation, a central problem of Carnap's account is that it remains stuck in a "lingua-centric predicament". That is, Carnap's designation rules fail to connect names and predicates to extra-linguistic objects or properties (Hall 1944). For this reason, Hall suggests that Carnap's semantic rules should be complemented by "empirical ties" that establish such a connection between a language and the world. Olen points out that Bergmann, in contrast to Hall, embraces such a formalistic reading of Carnap's pure semantics. For Bergmann, pure semantics should be conceived of as a purely syntactic or metalinguistic analysis of semantic concepts. According to him, this is precisely what Carnap is doing in his book: rules of designation do not relate expressions of a language to their non-linguistic referents, but rather expressions with names of them (Bergmann 1944).

In the second part of the article, Olen gives a critical assessment of these two conflicting views by turning to a closer discussion of Carnap's work on designation rules in (Carnap 1942). He emphasizes the difference between pure and formalized semantics and argues, contra both Bergmann and Hall, that Carnap's account of pure semantics is not formal in the sense that it abstracts from the 'extra-linguistic reference' of the expressions in question. For Carnap, rules of designation determine a relationship between the expressions of an object language and non-linguistic objects, properties, and propositions. As Olen shows, the main reason for Hall's and Bergmann's misreading of Introduction to Semantics lies in the fact that they interpret Carnap's pure semantics as a formalized or purely syntactical study of language in the above sense.

The final part of the paper retraces the influence of Hall's and Bergmann's interpretations (that is, the "Iowa reading") of Carnap's semantics on subsequent philosophy in the US, in particular on Wilfried Sellar's work on pure pragmatics from the 1940s. Olen argues here that Sellars' work is directly motivated by the Iowa interpretation of 
Carnap's pure semantics as a merely syntactical, metalinguistic study of languages. More specifically, Sellar's pure pragmatics can be viewed as the attempt to complement this formal study of languages with a pragmatic study of semantic designation that explains how expressions are connected to their non-linguistic designata.

\section{Tolerance, rationality, and the normativity of logic}

Two articles in the volume discuss a central topic in Carnap's philosophy of logic, namely his principle of tolerance first stated in Carnap (1934). Florian Steinberger's "Frege and Carnap on the normativity of logic" focuses on Carnap's understanding of the normative status of logic in light of his logical conventionalism. In particular, the article compares Carnap's conception of logic with that of his teacher Frege. Steinberger starts by discussing two important aspects of Frege's own views on the normativity of logic, namely the questions why logic is normative and, secondly, in what sense it is normative to him. Concerning the first point, Steinberger argues that according to Frege, laws of logic are primarily descriptive laws, i.e. laws stating general regularities about reality. But how can such descriptive laws be normative? As Steinberger points out, for Frege, the descriptive adequacy of a logical law "issues prescriptions" about the correct account of reasoning. Thus, the normativity of logic is grounded in its descriptive adequacy for Frege. Concerning the second question, Steinberger introduces a Searlean distinction between a regulative and a constitutive understanding of norms and argues that, at least for Frege, logical norms are constitutive for reasoning (similarly to the way in which the rules of chess are constitutive for the game). Steinberger calls this the "constitutivity thesis" in Frege's account of logic.

The second thematic part of the paper focuses on Carnap's account of the normative status of logic. As is well known, Carnap rejects both Frege's logical absolutism and his logical realism, that is, the idea that there exists a logic that correctly describes reality. Instead, the principle of tolerance implies a logical pluralism according to which any logical system can be adopted for the analysis of mathematics and the natural sciences. The justification of such systems is not to be based, as in Frege's understanding, on criteria of external correctness or descriptive adequacy, but rather on purely pragmatic considerations. As a consequence of this, Steinberger argues, Carnap's view is best described as a voluntarist account of logical norms: by choosing one logical system over another, we deliberately adopt norms of reasoning that will guide our thinking. Thus, according to Carnap's voluntarism, logical normativity is essentially self-imposed.

In spite of the differences to Frege's logical realism, Steinberger argues that there is a strong continuity between Carnap's account and the Fregean picture on how logic is normative. In particular, the general assumption that logic is providing constitutive norms for reasoning is also present Carnap's understanding of logic. In Carnap's case, however, such norms are not constitutive for reasoning in general, but only for forms of scientific inquiry. Steinberger calls this the "relativized constituitivity 
thesis". Roughly put, this is the view that different logical systems or, in Carnap's terminology different 'linguistic frameworks' can be constitutive for a scientific inquiry in the sense of setting standards of correct reasoning for the science in question. Given this relativized account of logical normativity, Steinberger finally discusses the question whether Carnap's logical tolerance does not commit him to the assumption of a core logic that underlies all linguistic forms.

André Carus' "Carnapian rationality" also focuses on the principle of tolerance, but from a different angle. The article investigates whether Carnap's principle is compatible with a wider conception of rationality. Carus starts his discussion with a survey of Carnap's mature work on the logical foundations of probability, in particular on the assumption of the uniformity of nature over time and its role in justifying inductive inferences. If this assertion is understood as a synthetic statement, then it has to be justified inductively and thus cannot provide a foundation for induction. However, according to Carnap, probable uniformity presents a purely analytic statement of logical probability. The rationality of the inductive method is thus not undermined by this assumption, given its analytic character.

As Carus points out, several objections have been raised against this argument in post-Quinean empiricist philosophy. According to the one objection present in work by John Earman, the notions of analyticity and aprioricity used by Carnap in the justification of the rationality of the inductive method are problematic since they are often used in connection with the notion of "inductive intuition". For instance, in the Schilpp volume (1963), Carnap argues that the justification of axioms of inductive logic can be given in terms of "intuitive judgements concerning inductive validity, i.e., concerning inductive rationality of practical decisions". The same notion of "inductive intuition" also figures prominently in Carnap's talk at the LSE conference in 1965.

Carus' main focus in his article is to further clarify Carnap's use of these notions in the justification of inductive logic and to defend it against the criticism raised by Popper, Earman, and others. As Carus points out, rationality for Carnap means a choice of a specific logic, both inductive and deductive, in a given theoretical context. Decisions concerning the choice of such a logic, i.e. "decisions about rationality", are not merely based on purely pragmatic considerations in Carnap's mature work, but are more principled. As Carus puts it, they are "more deeply rooted in scenarios of practical rationality". Nevertheless, both deductive and inductive reasoning as forms of rationality are decidedly analytic and a priori for Carnap. Given this, what precisely does he mean by inductive intuition? According to Carus, Carnapian intuition should be understood as trained instinct, that is, as a kind of "first-hand" experience that allows experts to clarify the informal content of a concept in the process of explication. (A closer discussion of Carnap's method of explication is given in the article by Dutilh-Novaes \& Reck discussed below.)

The second part of the article addresses a different objection, namely that Carnap's account of rationality can only amount to a form of instrumental rationality as opposed to a kind of value rationality. This concerns also the fact that rational decisions are always framework-relative, that is, internal to a given linguistic framework for him. In 
turn, decisions about the choice of such frameworks and meta-theoretical reasoning about them more generally can thus not be rational. Carus shows that the relationship between theoretical (i.e. framework-dependent) knowledge and the realm of practical decisions was a dialectical one for Carnap: theoretical reasoning shapes our set of admissable practical decisions. In turn, practical considerations determine the choice of linguistic frameworks and thus the chosen form of rationality. ${ }^{7}$ Carus' article concludes with a general outline of a Carnapian rationality that balances between the two objections discussed here, namely the post-Quinean objection regarding the existence of external questions and the post-Kantian objection that Carnap's external viewpoint is too weak to provide a proper foundation of moral principles.

The special issue also contains a transcribed and translated version of Carnap's so far unpublished notes "Value concepts (1958)" preceded by a short introduction by A. W. Carus. The notes were originally written in 1958 as a part of Carnap's reply to A. Kaplan's essay "Logical empiricism and value judgments" in the 1963 Schilpp volume, but were eventually not included in the book. Carnap's published reply contains a suggestion how to formalize value concepts and presents his only substantial contribution to the logic of normative statements. The notes contained in this special issue make a number of important additions to his published reply. In particular, Carnap distinguishes here between two kinds of value functions, namely partial and more comprehensive moral value functions that are also discussed in detail in Carus' research article. Carnap further presents here a definition of "relative rationality" of an agent's actions based on a set of possible actions, a body of evidence, and a partial value function.

Carnap further addresses the question whether there are standards of rationality for partial value functions in his notes. He argues that while inductive logic and factual knowledge is irrelevant in this respect, there may be "purely valuational criteria" that allow one to select between such functions. A number of possible formal criteria are suggested here. Carnap finally proposes a definition for an agent to act perfectly rational in a certain time period based on the notion of relative rationality and with respect to a given value function and a credence function.

\section{Explication, empirical significance, and logic of science}

Two articles contained in this special issue deal with Carnap's contributions to the logical formalization of philosophical concepts and its methodology. Catarina Dutilh Novaes' and Erich Reck's “Carnapian explication, formalisms as cognitive tools, and the paradox of adequate formalization" focuses on a key concept in Carnap's methodology of scientific philosophy, namely the notion of logical explication. The authors give a comparative discussion of Carnap's method of explication as a way to formalize pre-theoretical concepts in the sciences and Dutilh Novaes' account of "formalisms as cognitive tools" developed in her recent book Formal Languages in

\footnotetext{
${ }^{7}$ Compare Carus (2007) for a more detailed study of this topic in Carnap's work.
} 
Logic (Dutilh Novaes 2012). The paper has three thematic parts. In the first part, Carnap's account of explication (as developed in Carnap (1947) and, more extensively, in Carnap (1950)) is compared to Alfred Tarski's discussion of the "material adequacy" of logical formalizations in his work on truth and logical consequence (Tarski 1986). For Carnap, a successful explication is the act of replacing an informal concept by a precise one that meets four conditions, namely similarity, fruitfulness, exactness, and simplicity. Dutilh Novaes and Reck give a closer discussion of Carnap's understanding of each of these criteria of explication as well as his philosophical motivations for each.

In the second part of the article, the authors further illustrate Carnap's adequacy conditions of explication by turning to so-called statistical prediction rules (SPRs), that is, algorithms formulated on the basis of given statistical data. As is shown in the cases of medical diagnostics and the estimation of future wine prices, such formal tools often outperform expert intuition-based predictions given the fact that cognitive biases or other non-relevant factors are simply ruled out in them. How are SPRs related to Carnapian explication? While the formulation of such rules based on given statistical data is not generally a case of explication since no concept formation is involved, the authors argue that there is nevertheless an important link to Carnap's method. In particular, two of Carnap's desiderata, namely exactness and fruitfulness, also play a central role in the formulation of such rules. SPRs can be understood as cases where precisification leads to theoretical fruitfulness. In the present context, this does not mean the formulation of new results or theorems, but the fact that one yields more accurate predictions, for instance, refined diagnoses as in the medical example discussed in the article.

The final part of the article contains a discussion of what Dutilh Novaes and Reck call the paradox of adequate formalization (a version of the well-known 'paradox of analysis'). In the context of Carnap's method, this is a fundamental tension between two of his criteria for successful explication, namely similarity and theoretical fruitfulness. Roughly put, the fact that a logical explication should allow one to gain novel or refined theoretical results or make new discoveries is in direct conflict with the assumption that the explicatum should be as similar as possible to the inexact explicandum. More precisely, the authors argue that the more theoretically fruitful an explication is, the less similar the formal concept usually is to the pre-theoretic one. However, given that fruitfulness is the main adequacy condition in Carnapian explication and thus more important than conceptual similarity, this tension does not pose a crucial problem for his account of logical formalization.

Sebastian Lutz's “Carnap on empirical significance” investigates a particular case of Carnapian explication in the philosophy of science. The article surveys different proposals in Carnap's work on how to formalize the notion of empirical significance of scientific statements and scientific terms. Lutz argues that while Carnap's early attempts to provide such an account fails, he did in fact present a successful criterion of significance for sentences in his mature work on the logic of science. Lutz's analysis distinguishes between Carnap's early work in Europe (up to late 1930s), in which significance is based on the idea of reducibility of scientific expressions to 
basic terms, and his subsequent work in the US where this idea is eventually given up. Concerning the first period, it is shown that Carnap offers different versions of four technical notions that should provide a criterion for significance for statements, namely translatability, falsifability, and verifiability as well as confirmability (based on inductive inferences). A central focus of Lutz's article is to show how these different notions (as understood in Carnap's work from the 1930s) are related. Specifically, it is shown that when inductive inferences are treated probabilistically, falsifiability entails confirmability and verifiability entails disconfirmability. However, contra Carnap's suggestion in "Testability and meaning" (Carnap 1936), confirmability and translatability are shown to be non-equivalent by Lutz.

The second part of the article investigates Carnap's work on empirical significance after his emigration to the United States, beginning with his Foundations of Logic and Mathematics (Carnap 1939). Concerning the significance of terms, Carnap outlines two methods of relating abstract (or theoretical) to primitive (or observational) terms here. According to a bottom-up approach, primitive terms are introduced first. Abstract terms are then introduced in terms of reduction sentences. According to a top-down approach, abstract terms are implicitly defined by the axioms of a scientific theory in question. They are interpreted only partially however, based on the given empirical interpretation of the primitive terms. This method of partial interpretation presents, as Lutz shows, a significant shift from Carnap's earlier empiricist view that scientific terms should be directly reducible to observational terms. Lutz provides a detailed model-theoretic analysis of Carnap's alternative criteria for the significance of theoretical terms. He also discusses their relation to previous attempts of formulating such a criterion in terms of direct reducibility to observational terms.

Regarding the notion of empirical significance of statements, Lutz shows that Carnap's different explications based on the notions of translatability, verifiability, falsifiability, and non-trivial content turn out to be successful if his mature views on logical theory reconstruction are taken into account. In particular, given Carnap's understanding of the Ramsey sentence and Carnap sentence of a theory as expressing its synthetic and analytic content respectively, it follows that the different criteria are in fact equivalent (at least if attention is restricted to deductive inferences). The paper concludes with a number of more general observations on the different explications of empirical significance in Carnap's work.

\footnotetext{
Acknowledgements As the editor of this special issue, I would like to thank the participants of the "Carnap on Logic" conference for their contributions and the stimulating discussions during the event. The conference was generously funded by the German Research Foundation (grant number SCHI 1269/1-1). I am also grateful to the numerous reviewers for their efforts to improve the articles contained in this volume. Finally, I would like to thank Hannes Leitgeb, André Carus, Erich Reck, John Wigglesworth, and Steve Awodey for their advice and for helpful discussions on this special issue and the preceding conference. Work on this editorial introduction was partly funded by the Austrian Science Fund (grant number P27718-G16).
} 


\section{References}

Awodey S, Carus A (2001) Carnap, completeness, and categoricity: the Gabelbarkeitssatz of 1928. Erkenntnis $54: 145-172$

Bergmann G (1944) Pure semantics, sentences, and propositions. Mind 53:238-257

Bonnay D (2009) Carnap's criterion of logicality. In: Wagner P (ed) Carnap's Logical Syntax of Language, Houndsmills, Basingstoke: Palgrave Macmillan, pp 147-164

Carnap R (1927) Eigentliche und uneigentliche Begriffe. Symposion: Philosophische Zeitschrift für Forschung und Aussprache 1(4):355-374

Carnap R (1928) Der logische Aufbau der Welt. Weltkreis-Verlag, Berlin-Schlachtensee

Carnap R (1928/2000) Untersuchungen zur allgemeinen Axiomatik. Wissenschaftliche Buchgesellschaft, Darmstadt

Carnap R (1934) Logische Syntax der Sprache. Schriften zur wissenschaftlichen Weltauffassung, vol. 8, Wien: Springer

Carnap R (1936) Testability and meaning. Philosophy of Science 3(4):420-468

Carnap R (1939) Foundations of Logic and Mathematics. Chicago: University of Chicago Press

Carnap R (1942) Introduction to Semantics. Cambridge: Harvard University Press

Carnap R (1947) Meaning and Necessity. Chicago: University of Chicago Press

Carnap R (1950) Logical Foundations of Probability. Chicago: University of Chicago Press

Carnap R (1963) Replies and systematic expositions. In: Schilpp P (ed) The philosophy of Rudolf Carnap, LaSalle, IL: Open Court, pp 859-1013

Carus AW (2007) Carnap and Twentieth-Century Thought: Explication as Enlightenment. Cambridge University Press, New York

Coffa A (1991) The Semantic Tradition from Kant to Carnap. New York: Cambridge University Press

Dutilh Novaes C (2012) Formal Languages in Logic. Cambridge: Cambridge University Press

Friedman M (1999) Reconsidering logical positivism. Cambridge: Cambridge University Press

Friedman M, Creath R (eds) (2007) The Cambridge Companion to Carnap. Cambridge: Cambridge University Press

Frost-Arnold G (2013) Carnap, Tarski, and Quine at Harvard. Conversations on logic, mathematics, and science. Chicago: Open Court

Goldfarb W (1979) Logic in the twenties: the nature of the quantifier. Journal of Symbolic Logic 44:351368

Hall E (1944) The extra-linguistic reference of language (ii.). Mind 53:25-47

van Heijenoort J (ed) (1967) From Frege to Gödel. A Source Book in Mathematical Logic, 1897-1931. Harvard University Press, Cambridge, Mass.

Hintikka J (1992) Carnap's work in the foundations of logic and mathematics in a historical perspective. Synthese 93:167-189

Mac Lane S (1938) Carnap on logical syntax. Bulletin of the American Mathematical Society 44:171-176 Quine WV (1960) Carnap and logical truth. Synthese 12(4):350-374

Reck E (2004) From Frege and Russell to Carnap: Logic and logicism in the 1920s. In: Awodey S, Klein C (eds) Carnap Brought Home: The View from Jena, Open Court, La Salle, IL, pp 151-180

Tarski A (1935) Der Wahrheitsbegriff in den formalisierten Sprachen. Studia Philosophica 1:261-405

Tarski A (1936) Über den Begriff der logischen Folgerung. Actes du Congrés International de Philosophie Scientifique 7:1-11, translated in Tarski (2002)

Tarski A (1986) What are logical notions? History and philosophy of logic 7:143-154

Tarski A (2002) On the concept of following logically. History and Philosophy of Logic 23(3):155-196

Wagner P (ed) (2009) Carnap's Logical Syntax of Language. Palgrave Macmillan 